\title{
Definitive re-irradiation of locally recurrent esophageal cancer after trimodality therapy in patients with a poor performance status
}

\author{
DANIEL W. KIM ${ }^{1}$, SANA RAOOF ${ }^{2}$, NAYAN LAMBA ${ }^{1}$, GRACE LEE ${ }^{2}$, DANIELLE S. BITTERMAN ${ }^{1}$, \\ AMANDEEP R. MAHAL ${ }^{3}$, NINA N. SANFORD ${ }^{4}$, MIRANDA B. LAM ${ }^{1}$ and HARVEY J. MAMON ${ }^{1}$ \\ ${ }^{1}$ Department of Radiation Oncology, Dana-Farber Cancer Institute/Brigham and Women's Hospital; \\ ${ }^{2}$ Harvard Medical School, Boston, MA 02115; ${ }^{3}$ Yale School of Medicine, New Haven, CT 06510; \\ ${ }^{4}$ Department of Radiation Oncology, University of Texas Southwestern Medical Center, Dallas, TX 75390, USA
}

Received January 15, 2020; Accepted April 27, 2020

DOI: $10.3892 / \mathrm{mco} .2020 .2044$

\begin{abstract}
There are few treatment guidelines for locally recurrent esophageal cancer after trimodality treatment (pre-operative chemoradiation followed by surgery) in patients with a poor performance status. The purpose of this single institutional, retrospective study was to evaluate the clinical outcomes and toxicities of definitive-intent re-irradiation for patients with recurrent esophageal cancer with a poor performance status [ECOG (Eastern Cooperative Oncology Group) 22]. Seven patients were identified with a median age of 74 years (range, 61-81 years). Four patients were ECOG 2 and three patients were ECOG 3. The median follow-up time after re-irradiation was 49 months. The median interval between initial radiotherapy and re-treatment was 32 months. Six patients received concurrent chemotherapy [carboplatin + paclitaxel in three patients; folinic acid, fluorouracil, oxaliplatin $($ FOLFOX $)+5$-fluorouracil in one patient; FOLFOX in one patient, and capecitabine in one patient]. At the last follow-up, the six patients who underwent concurrent chemotherapy had stable disease (86\%), while the one who did not receive chemotherapy progressed (14\%).
\end{abstract}

Correspondence to: Dr Harvey J. Mamon, Department of Radiation Oncology, Dana-Farber Cancer Institute/Brigham and Women's Hospital, 75 Francis Street, Boston, MA 02115, USA

E-mail: hmamon@bwh.harvard.edu

Abbreviations: RT, radiotherapy; ECOG, Eastern Cooperative Oncology Group; GE, gastroesophageal; 4D, 4-dimensional; CT, computed tomography; PET, positron emission tomography; GTV, gross tumor volume; CTV, clinical tumor volume; ITV, internal target volume; IMRT, intensity modulated RT; 3D, 3-dimensional; CTCAE, Common Terminology Criteria for Adverse Events; FOLFOX, folinic acid + fluorouracil + oxaliplatin; 5-FU, 5-fluorouracil; BED, biologically effective dose; KPS, Karnofsky performance status

Key words: esophageal cancer, re-irradiation, poor performance status, chemoradiation, recurrent
Two patients developed metastases. Three patients developed acute ( $<6$ months) grade 4 toxicities (dysphagia, anemia, esophagitis). There were no early deaths attributable to treatment. Late toxicities (>6 months) were limited to grades 1 and 2 dysphagia and pneumonitis in four patients. In conclusion, definitive re-irradiation of recurrent esophageal cancer in patients with a poor performance status appears to be safe with acceptable acute toxicity and late complications. It also appears to result in durable local control when combined with chemotherapy, albeit with a small number of patients and limited follow-up.

\section{Introduction}

Esophageal cancer is the eighth most common cancer and is the sixth leading cause of cancer deaths worldwide (1). Greater than 450,000 new cases of esophageal cancer are reported worldwide every year, with its incidence rising (1). In non-metastatic, locally advanced disease, trimodality treatment, which includes pre-operative chemoradiation followed by surgery, is the standard of care.

However, over $50 \%$ of patients with esophageal cancer treated with pre-operative chemoradiation followed by surgery experience local or regional recurrence (1). In patients with locoregional recurrence, surgery, when feasible, is the standard for definitive local control, with salvage esophagectomy offering a 5-year survival rate of $25 \%$ (1). However, salvage surgery is associated with high morbidity and is typically limited to patients with a good performance status.

The management of patients with a poor performance status who experience locoregional esophageal recurrence after trimodality therapy remains challenging. Many of these patients often receive palliative RT to treat local symptoms only. To date, only a few series have explored definitive, non-palliative re-irradiation for locally recurrent esophageal cancer (2-4). These studies have demonstrated that re-irradiation in such cases may be effective but with variable toxicity. However, they have excluded patients with a poor performance status [Eastern Cooperative Oncology Group $(\mathrm{ECOG}) \geq 2$ ] 
We hypothesized that definitive-intent re-irradiation may offer durable local control with acceptable toxicities even in patients with a poor performance status. Thus, in the present study, we sought to evaluate the role of definitive re-irradiation for recurrent locoregional esophageal cancer after trimodality therapy in patients with a poor performance status.

\section{Materials and methods}

Subjects. The inclusion criteria for this retrospective analysis were as follows: i) Biopsy-proven localized recurrence of cancer of the esophagus or gastroesophageal (GE) junction; ii) history of pre-operative radiation followed by surgery; iii) non-metastatic disease; iv) receipt of non-palliative re-irradiation dose of $>45 \mathrm{~Gy}$, and v) ECOG $\geq 2$. The exclusion criteria were everything outside of the categories listed in the inclusion criteria. Baseline clinical data of the subjects were collected including age, sex, ECOG performance score, primary site of disease, clinical TNM stage, dose of radiotherapy (RT) received, RT technique, chemotherapy used, histology, and pathologic TNM. Not all patients had their initial pre-operative chemoradiation and/or surgery at our institution. All patients had their re-irradiation courses at our institution.

The present study was approved by the Dana-Farber Cancer Institute Institutional Review Board (approval no. 18-381). Patients who participated in this research had complete clinical data. The signed informed consents were obtained from the patients or the guardians.

Re-irradiation treatment. All patients underwent 4-dimensional (4D) computed tomography (CT) simulation. Positron emission tomography (PET) scans, when available, were fused with the simulation scan in order to aid in target delineation. Gross tumor volume (GTV) included macroscopic tumor and enlarged lymph nodes. Clinical tumor volume (CTV) margin included the GTV with $2-3 \mathrm{~cm}$ proximal and distal borders. The radial borders were $0.7-1 \mathrm{~cm}$ from the GTV. The expansion into cardiac tissue was limited to $0.5 \mathrm{~cm}$ when the GTV was located at the esophagus-heart interface. The CTV did not include elective uninvolved nodes in the CTV for the re-irradiation course. An internal target volume (ITV) was created to account for tumor motion. The CTV to planning target volume (PTV) margin was $0.5-1 \mathrm{~cm}$. Cone-beam CT was performed pre-treatment. All re-irradiation was performed with intensity modulated RT (IMRT), whereas initial RT was predominantly 3-dimensional (3D) conformal with only two patients receiving IMRT.

Statistical analysis. Kaplan-Meier survival curves were generated from the time of re-irradiation of the recurrence to the event point of interest. The events were defined as local failure, metastasis, and any progression. Toxicities were graded based on Common Terminology Criteria for Adverse Events (CTCAE) version 5.0. Toxicity was categorized as either acute (during or within 3 months after re-irradiation) or late (over 3 months after completion of re-irradiation). Stata Statistical Software (StataCorp) was used to perform all statistical analyses.

\section{Results}

Patient, tumor and treatment characteristics. A total of ten patients met the eligibility criteria. Seven patients were included in this study as three patients did not have adequate clinical information available. The median interval between initial RT and re-irradiation was 32 months. Patient characteristics at initial treatment and re-irradiation are summarized in Tables I and II, respectively. There were six males and one female. Pathologic stage following their initial chemoradiation course was I $(n=3)$, II $(n=2)$, or III $(n=2)$. Primary histology in six patients was adenocarcinoma while one patient had squamous cell histology. Tumor locations included GE junction $(n=3)$, lower esophagus $(n=2)$, mid-esophagus $(n=1)$, and upper esophagus $(n=1)$. The initial radiation dose ranged from 45 to $64.8 \mathrm{~Gy}$ and the re-irradiation dose ranged from 50.4 to $68.4 \mathrm{~Gy}$. ECOG performance status at the time of initial RT was $0 \quad(n=4)$ or $1(n=3)$. ECOG performance status at re-irradiation was $2(n=6)$ or $3(n=1)$. Three of the seven recurrences overlapped with the prior RT field. The median interval between radiation treatments was 33 months (range 8.6-57.4 months). All seven patients had initial concurrent chemotherapy with carboplatin and paclitaxel. At time of re-irradiation, six had concurrent chemotherapy during re-irradiation (with either carboplatin and paclitaxel, FOLFOX (folinic acid, fluorouracil, oxaliplatin) +/- 5-FU (5-fluorouracil), or capecitabine) and one patient received RT alone. The patient who received RT alone was thought to be unable to tolerate concurrent chemotherapy.

Disease control. Median follow-up time after re-irradiation was 49 months. Local failure-free survival, metastasis-free survival, and progression free-survival data are summarized in Figs. 1-3, respectively. Progression free-survival was defined as the interval to any recurrence, local failure or metastasis. At last follow-up, all patients except one achieved stable disease with re-irradiation; progressive disease was observed in the one patient who did not receive concurrent chemotherapy.

Toxicity. Toxicities from treatment are summarized in Table III. All patients suffered from at least grade 1-2 toxicities after re-irradiation with fatigue being the most common. Five patients had grade 3 toxicity, including esophagitis $(n=3)$, neutropenia $(n=1)$, dysphagia $(n=2)$, and dermatitis $(n=1)$. Three patients had grade 4 toxicities: One patient each with dysphagia, anemia, and esophagitis. There were no grade 5 toxicities seen after re-irradiation. Four patients had late toxicities of dysphagia or pneumonitis but all were low grade in severity (grades 1 and 2).

Re-irradiation planning. The planning parameters are shown in Table IV. The PTV was planned to ensure $100 \%$ of the PTV received at least $95 \%$ of the prescription dose. Maximum dose heterogeneity was set at $107 \%$. Biologically effective dose (BED) with an $\alpha / \beta$ ratio of 2 for spinal cord was used to calculate total cumulative dose. Maximum cumulative BED for the spinal cord was set at $<135 \mathrm{~Gy}$ with all re-irradiation intervals being longer than 6 months $(5,6)$. The PTV coverage at $95 \%$ of 


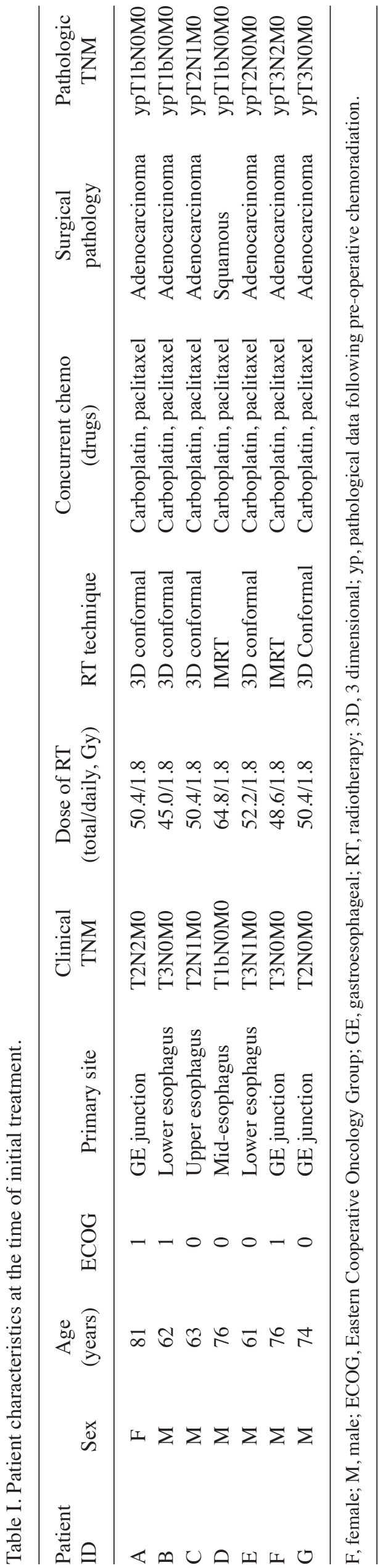

prescription dose ranged from 96.1 to $100 \%$ (Table IV). The maximum dose given to spinal cord ranged from 10.9 to 47.8 Gy. Heart V25 ranged from 2.2 to $9.4 \%$ and lung V20 ranged from 4 to $22.7 \%$.

\section{Discussion}

Previous studies exploring the use of re-irradiation in patients with locoregional recurrence of esophageal cancer have suggested that non-palliative treatment may be a safe and effective strategy for prolonging overall survival in patients with a good performance status (ECOG 0 or 1$)(4,7)$. We examined the generalizability of these findings in patients with poor performance status $(E C O G \geq 2)$ and found that definitive, non-palliative re-irradiation may offer durable local control with acceptable toxicity in this patient population as well. Although our study was comprised of a small cohort, to our knowledge, this represents the first study to specifically assess the role of re-irradiation in patients with locoregional recurrence of esophageal cancer with a poor performance status.

Chen et al (2) compared the efficacy of salvage surgery versus re-irradiation in patients with recurrent esophageal cancer and good performance status (ECOG 0 or 1), finding no difference in survival but more complications (esophageal fistula/perforation) in the re-irradiation group. A study of six patients re-irradiated in Japan for recurrent squamous cell esophageal cancer found a median survival time of 13.6 months (1.9-33.3 months) after re-irradiation, with three of six patients remaining alive at one year after re-irradiation (7). Out of six patients included in this study, one patient developed grade 4 leukopenia, and two patients developed grade 3 leukopenia. The authors compare their reported median survival time (13.6 months) to the expected survival time of patients with advanced esophageal cancer treated only with chemotherapy (9.9 months) (8).

A Korean study with short follow-up examined ten patients who were re-irradiated for recurrent esophageal squamous cell carcinoma and followed patients for a median of 4.9 months (3). These patients were either inoperable due to the extent of recurrent disease or they refused salvage surgery. At three months, this study observed two complete responses, one partial response, two stable disease states, and five cases of progression. Three patients developed grade 5 tracheoesophageal fistula; two of these three patients were re-irradiated within one year of the original radiation treatment. This small study identified several factors that may have played a role in the differing outcomes of the ten patients, including the total dose of re-irradiation (higher dose was associated with more severe toxicity), time interval between primary RT and re-irradiation (shorter interval was associated with more severe toxicity), extent of disease progression (disease progression may be confused with treatment-related toxicity), treatment modality, concurrent versus sequential chemotherapy, tumor stage, and irradiated volume of esophagus. Due to its limited size and short follow-up time, further clinical studies are required to understand the effects of each variable on re-irradiation safety and efficacy.

A larger Japanese study examined 237 patients receiving re-irradiation for oligo-lymph node recurrent (1-5 lymph 
Table II. Patient characteristics at re-irradiation.

\begin{tabular}{lcccccl}
\hline $\begin{array}{l}\text { Patient } \\
\text { ID }\end{array}$ & ECOG & $\begin{array}{c}\text { Overlap with } \\
\text { prior RT field? }\end{array}$ & $\begin{array}{c}\text { Dose of RT } \\
\text { (total/daily, Gy) }\end{array}$ & $\begin{array}{c}\text { Interval between RT } \\
\text { treatment, months }\end{array}$ & $\begin{array}{c}\text { RT } \\
\text { technique }\end{array}$ & $\begin{array}{c}\text { Concurrent chemo } \\
\text { (drugs) }\end{array}$ \\
\hline A & 3 & Yes & $50.4 / 1.8$ & 27.6 & IMRT & None \\
B & 2 & No & $54.0 / 1.8$ & 57.4 & IMRT & FOLFOX, 5-FU \\
C & 2 & No & $59.4 / 1.8$ & 56.3 & IMRT & FOLFOX \\
D & 2 & Yes & $68.4 / 1.8$ & 11.0 & IMRT & Carboplatin, paclitaxel \\
E & 2 & No & $60.0 / 2$ & 32.7 & IMRT & Carboplatin, paclitaxel \\
F & 2 & Yes & $54.0 / 2$ & 8.6 & IMRT & Capecitabine \\
G & 2 & No & $59.4 / 1.8$ & 33.0 & IMRT & Carboplatin, paclitaxel \\
\hline
\end{tabular}

ECOG, Eastern Cooperative Oncology Group; RT, radiotherapy; IMRT, intensity modulated RT; FOLFOX, folinic acid, fluorouracil, oxaliplatin; 5-FU, fluorouracil.

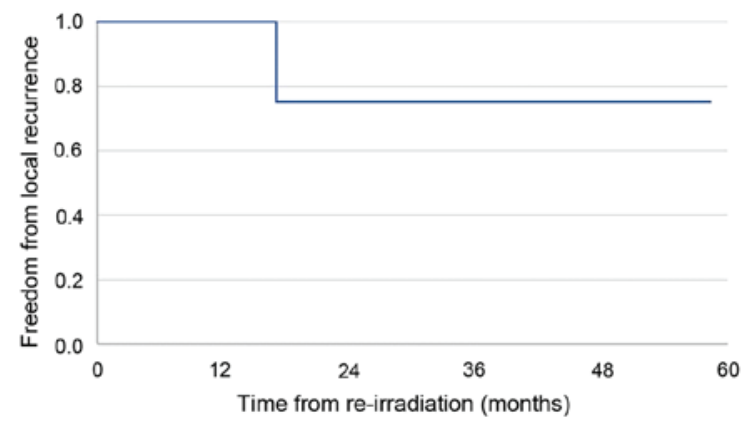

Figure 1. Local failure-free survival for patients $(n=7)$ with recurrent esophageal cancer and a poor performance status who received definitive re-irradiation.

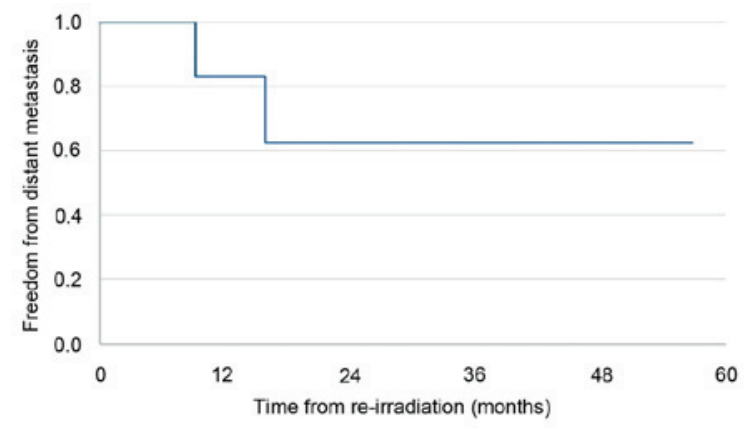

Figure 2. Metastasis-free survival for patients $(n=7)$ with recurrent esophageal cancer and a poor performance status who received definitive re-irradiation.

nodes positive) esophageal cancer over a 29.6-month follow-up period (4). The study included $83 / 237$ patients with Karnofsky performance status (KPS) $<90$. Over $97 \%$ of patients had squamous cell tumor histology. In this study, median survival time was 21.6 months; the 3-year overall survival was $37 \%$, local control was $45 \%$, progression-free survival was $24 \%$, and esophageal cancer-specific survival was $42 \%$. The 3-year overall survival in the KPS 80-100 group was 43.1\%, compared to $11.9 \%$ for KPS $\leq 70$. This large study identified several positive prognostic factors for patients undergoing re-irradiation: Combined chemotherapy, disease-free interval $\geq 12$ months, max lymph node diameter $\leq 22 \mathrm{~mm}$, and

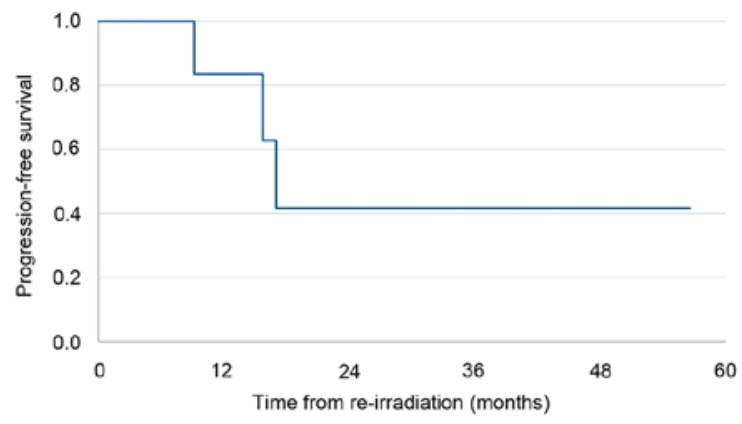

Figure 3. Progression-free survival for patients $(n=7)$ with recurrent esophageal cancer and a poor performance status who received definitive re-irradiation.

KPS $\geq 80 \%$. This study was adequately powered to support the feasibility of safe and beneficial usage of re-irradiation for recurrent esophageal cancer with oligo-lymph node recurrence. We provide a summary of the aforementioned studies in Table SI.

Esophageal adenocarcinoma and squamous cell carcinoma may differ in cancer cell biology, location within the esophagus, and sensitivity to RT. Conclusions drawn from re-irradiation of one tumor histology therefore may not apply to other histological types. Of note, each of the three aforementioned studies of re-irradiation in recurrent esophageal cancer include a predominance of patients with squamous cell histology. In our study, six patients had adenocarcinoma and only one had squamous histology, offering novel insight into the role of re-irradiation in recurrent adenocarcinoma of the esophagus.

To our knowledge, the present study is the first to specifically assess re-irradiation in patients with a poor performance status who have locally recurrent esophageal cancer after trimodality therapy. The patients in this study had a good performance status at the time of initial RT but a poor performance status (ECOG 2 or 3 ) at the time of re-irradiation. Over the 49 month median follow-up period, there were no patient deaths and only one case of progressive disease among seven patients treated with definitive re-irradiation. Six patients exhibited stable disease at last follow-up. The patient who progressed in this study was the only one who did not receive concurrent chemotherapy. 
Table III. Toxicity from re-irradiation.

\begin{tabular}{|c|c|c|c|c|c|}
\hline \multirow{2}{*}{$\begin{array}{l}\text { Patient } \\
\text { ID }\end{array}$} & \multicolumn{4}{|c|}{ Acute toxicity } & \multirow{2}{*}{$\begin{array}{l}\text { Late toxicity } \\
\text { grades } 1-5\end{array}$} \\
\hline & Grades 1 and 2 & Grade 3 & Grade 4 & Grade 5 & \\
\hline A & Fatigue, nausea & Esophagitis & - & - & Dysphagia (grade 1) \\
\hline $\mathrm{B}$ & Fatigue & Anemia, neutropenia & Dysphagia & - & Pneumonitis (grade 1) \\
\hline $\mathrm{C}$ & Anorexia & Esophagitis, dermatitis & Anemia & - & - \\
\hline $\mathrm{D}$ & Fatigue, anemia & Dysphagia, nausea & - & - & Dysphagia (grade 2) \\
\hline $\mathrm{E}$ & Fatigue, anemia, dermatitis & - & - & - & $\begin{array}{l}\text { Dysphagia (grade } 2 \text { ), } \\
\text { pneumonitis (grade } 1 \text { ) }\end{array}$ \\
\hline $\mathrm{F}$ & Fatigue, dermatitis & Dysphagia, esophagitis & & - & - \\
\hline $\mathrm{G}$ & Anorexia, anemia & - & Esophagitis & - & - \\
\hline
\end{tabular}

Table IV. Dose volume histogram from re-irradiation course.

\begin{tabular}{lcccrr}
\hline Patient ID & PTV @ 95\% of Rx, \% & Cord max, Gy & Heart V25, \% & Lung V5, \% & Lung V20, \% \\
\hline A & 100.0 & 10.9 & 5.5 & 12.8 & 4.0 \\
B & 96.8 & 33.4 & 2.2 & 43.5 & 22.7 \\
C & 99.3 & 47.8 & 3.1 & 6.2 & 3.5 \\
D & 96.5 & 10.6 & 3.5 & 34.3 & 20.1 \\
E & 96.1 & 30.2 & 8.3 & 42.4 & 16.4 \\
F & 99.8 & 11.8 & 9.4 & 35.5 & 18.0 \\
G & 100.0 & 43.4 & & \\
\hline
\end{tabular}

PTV, planning target volume; Rx, prescription dose; PTV @ 95\% of Rx (\%), percent of the PTV receiving at least 95\% of the prescription dose; $\mathrm{Vx}, \%$, percent of volume of interest receiving $\mathrm{xGy}$.

The outcomes in our study are more favorable than those reported in previous studies examining the role of re-irradiation in patients with locoregional recurrence of esophageal cancer. Our findings should be taken with consideration of the following main distinctions between our study and previous studies: i) The majority of our patients had adenocarcinoma histology, whereas other studies had predominantly squamous cell patients; ii) our study focused on poor performance status patients, whereas other studies did not stratify for this criterion, and iii) all patients were treated at a major academic medical center with significant experience in complicated re-irradiation cases and tremendous oncologic care support (9). Furthermore, it should be noted that this study is limited by lack of baseline organ (i.e. liver, kidney) function data at time of recurrence/re-irradiation. We also lack data regarding any hematologic, hepatic, or renal toxicities related to treatment.

In conclusion, this series of patients suggests that even in the setting of poor performance status, definitive re-irradiation may be given safely and achieve durable response in patients with locally recurrent esophageal cancer after trimodality therapy.

\section{Acknowledgements}

Not applicable.

\section{Funding}

No funding was received.

\section{Availability of data and materials}

The datasets used and/or analyzed during the present study are available from the corresponding author on reasonable request.

\section{Authors' contributions}

DWK, MBL, and HJM conceived and designed the study. SR, ARM, NL and DSB conducted the literature search. SR, ARM, NL, DSB, GL, MBL and NNS obtained data and contributed to analysis. DWK and MBL led the data analysis. SR, ARM, NL, DSB and GL prepared the manuscript. All authors provided critical feedback, discussed the results and its implications, and contributed to the final manuscript. DWK and HJM supervised the project. All authors read and approved the final manuscript.

\section{Ethics approval and consent to participate}

The present study was approved by the Dana-Farber Cancer Institute Institutional Review Board (approval no. 18-381). 
Patients who participated in this research had complete clinical data. The signed informed consents were obtained from the patients or the guardians.

\section{Patient consent for publication}

Not applicable.

\section{Competing interests}

The authors declare that they have no competing interests.

\section{References}

1. Pennathur A, Gibson MK, Jobe BA and Luketich JD: Oesophageal carcinoma. Lancet 381: 400-412, 2013.

2. Chen Y, Lu Y, Wang Y, Yang H, Xia Y, Chen M, Song H, Li T, Li D, Wang J, et al: Comparison of salvage chemoradiation versus salvage surgery for recurrent esophageal squamous cell carcinoma after definitive radiochemotherapy or radiotherapy alone. Dis Esophagus 27: 134-140, 2013.

3. Kim YS, Lee CG, Kim KH, Kim T, Lee J, Cho Y and Koom WS: Re-irradiation of recurrent esophageal cancer after primary definitive radiotherapy. Radiat Oncol J 30: 182-188, 2012.

4. Yamashita H, Jingu K, Niibe Y, Katsui K, Matsumoto T, Nishina $\mathrm{T}$ and Terahara A: Definitive salvage radiation therapy and chemoradiation therapy for lymph node oligo-recurrence of esophageal cancer: A Japanese multi-institutional study of 237 patients. Radiat Oncol 12: 38, 2017.
5. Nieder C, Grosu AL, Andratschke NH and Molls M: Proposal of human spinal cord reirradiation dose based on collection of data from 40 patients. Int J Radiat Oncol Biol Phys 61: 851-855, 2005

6. Nieder C, Grosu AL, Andratschke NH and Molls M: Update of human spinal cord reirradiation tolerance based on additional data from 38 patients. Int J Radiat Oncol Biol Phys 66: 1446-1449, 2006.

7. Katano A, Yamashita $\mathrm{H}$ and Nakagawa $\mathrm{K}$ : Re-irradiation of locoregional esophageal cancer recurrence following definitive chemoradiotherapy: A report of 6 cases. Mol Clin Oncol 7: 681-686, 2017.

8. Cunningham D, Starling N, Rao S, Iveson T, Nicolson M, Coxon F, Middleton G, Daniel F, Oates J, Norman AR, et al: Capecitabine and oxaliplatin for advanced esophagogastric cancer. N Engl J Med 358: 36-46, 2008.

9. Lassig AA, Joseph AM, Lindgren BR, Fernandes P, Cooper S, Schotzko C, Khariwala S, Reynolds M and Yueh B: The effect of treating institution on outcomes in head and neck cancer. Otolaryngol Head Neck Surg 147: 1083-1092, 2012. International (CC BY-NC-ND 4.0) License. 